\title{
Prediction of Failure Risk for Underground Casing Pipe Based on Geostatistics
}

\author{
Yanhua Chen", a , Linlin Liü, b , Qingjie Zhu ${ }^{2, \mathrm{c}}$ and Yiliang Liü, \\ ${ }^{1}$ Earthquake Engineering Research Center, North China University of Science and Technology, \\ Tangshan 063210, Hebei Province, China \\ ${ }^{2}$ School of Petroleum Engineering, Changzhou University, Changzhou 213016, Jiangsu Province, \\ China \\ ${ }^{3}$ Department of Civil Engineering, North China Institute of Aerospace Technology, Langfang \\ 065000, Hebei Province, China \\ acy427@163.com, b974549693@qq.com, 'qjzhu@cczu.edu.cn, diuyiliang1987@163.com
}

Keywords: Risk prediction; Geostatistics; Underground pipe; Failure; Kriging interpolating.

Abstract. Failure analysis of underground casing pipe is very important for engineering protection. Failure of underground casing pipe is affected by many factors, and shows very strong spatial variability. Therefore, a predictive model is constructed based on geostatistics, in which spatial variability is taken into account. Through the spatial variability analysis of influence factors, interpolating images can be worked out with kriging and mathematical fitting techniques. As an application example, the failure risk is calculated by this model with interpolating images. The distribution of failure risk of underground casing pipe is investigated, and some advice is proposed.

\section{Introduction}

In recent years, there are many researches on the mechanism of underground casing pipe failure, and the failure risk analysis based on statistics becomes more and more important [1-3]. Ordinary, failure risk of underground casing pipe is affected by many factors, and shows very strong spatial variability $[4,5]$. In the predictive model, both criteria weights and order weights should be considered. Since the 20th century, many progresses have been made on weights calculation, and they are applied to many domains [6-9]. Geostatistics is the core of surface analysis, and interpolating images can be worked out by geostatistical techniques for the space sample data. Spatial variability with their values and locations of space sample points can be analyzed, and interpolating surfaces can be obtained with ordinary kriging and mathematical fitting techniques $[10,11]$.

In this article, the spatial variability is analyzed for influence factors, and interpolating images are worked out with kriging and mathematical fitting techniques. The failure risk is calculated with predictive model, and the distribution of failure risk of underground casing pipe is investigated.

\section{Risk Predictive Model}

If the number of space points is $m, i$ represents any one of those space points. The number of influence factors is $n$, and $j$ represents any one of those factors. Here, $x_{i j}$ is the $j$-th factor's value in any space point $i$. The failure risk is represented as $\mathrm{R}_{i}$, then, it can be calculated as follow,

$$
R_{i}=\sum_{j=1}^{n}\left(\frac{u_{j} v_{j}}{\sum_{j=1}^{n} u_{j} v_{j}}\right) x_{i j}
$$

In which, $\mathrm{u}_{j}$ is criteria weight of the factor $j, \mathrm{v}_{j}$ is order weight of the factor $j$.

Therefore, weights of failure risk are calculated from criteria weights and order weights. Image that the same factor in different space point has the same weight. It means that criteria weights only related 
to influence factor, does not relate with the space location. Ordinarily, a comparison matrix A is constructed as $\left[a_{i j}\right]$, criteria weights are written as,

$$
\begin{aligned}
& u_{j}=\frac{u_{j}}{\sum_{i=1}^{n} \overline{u_{i}}} \quad i, j=1,2, \mathrm{~L}, n \\
& \bar{u}_{i}=\sum_{j=1}^{n} \bar{a}_{i j} \quad i, j=1,2, \mathrm{~L}, n \\
& \bar{a}_{i j}=\frac{a_{i j}}{\sum_{k=1}^{n} a_{k j}} \quad i, j, k=1,2, \mathrm{~L}, n
\end{aligned}
$$

Before the calculation of order weighs, all factors' values in any space point " $i$ " need to be reordered from maximum to minimum. Order weights only relate to space location, and do not relate with the factor. The order weighs can be obtained as follow,

$$
v_{j}=\frac{n-r_{j}+1}{\sum_{l=1}^{n}\left(n-r_{l}+1\right)} \quad(j, l=1,2, \mathrm{~L} n)
$$

Eq. (5) is the basic rank order method to calculate order weights. In this method, if there are three factors, it means $n$ equals to 3, then the biggest factor in value is treated as $r_{1}$, and equals to 1 . And so on, $r_{2}$ equals to 2 , and $r_{3}$ is 3 . It means that the value of factors determine order weights.

Now, compute $x_{i j}$ in Eq. (1). For any influence factor " $j$ ", the normalization is written as,

$$
x_{j}=\frac{x_{j}-\operatorname{Min}\left(x_{j}\right)}{\operatorname{Max}\left(x_{j}\right)-\operatorname{Min}\left(x_{j}\right)}
$$

And for any space point " $i$ ", $x_{i j}$ is calculated from known neighbor known points, and written as,

$$
x_{i j}=\sum_{l=1}^{p} \lambda_{l} z_{l j} \quad l=1,2,3 \mathrm{~L} p
$$

In which, $\mathrm{z}_{l j}$ is the value of the $l$-th known point, and $p$ is the number of interpolating space points. $\lambda_{l}$ is weight of $z_{l j}$, according to ordinary kriging interpolation, it must meet the following conditions,

$$
\begin{aligned}
& \sum_{l=1}^{p} \lambda_{l}=1 \quad l=1,2,3 \mathrm{~L} p \\
& \sum_{l=1}^{p} \gamma_{(l-m)} \lambda_{l}+\mu=\gamma_{(i-m)} \quad m=1,2,3 \mathrm{~L} \quad p
\end{aligned}
$$

In which, $\mu$ is Lagrange parameters, $\gamma$ is semivariogram. The semivariogram can be used to describe spatial variability.

Thus, we can forecast the failure risk of underground casing pipe through above calculation based on the data of influence factors.

\section{Application Example}

As an example application, three factors in J607 block are adopted. Because there are too much data, some of them are extracted as table 1 . 
Table 1 Extracted data of influence factors

\begin{tabular}{|c|c|c|c|c|c|c|c|}
\hline No. & $\begin{array}{c}\text { Volume of steam } \\
\text { injection/m }\end{array}$ & $\begin{array}{c}\text { Volume of } \\
\text { production/m }\end{array}$ & $\begin{array}{c}\text { Times of } \\
\text { injection }\end{array}$ & No. & $\begin{array}{c}\text { Volume of steam } \\
\text { injection } / \mathrm{m}^{3}\end{array}$ & $\begin{array}{c}\text { Volume of } \\
\text { production/m }\end{array}$ & $\begin{array}{c}\text { Times of } \\
\text { injection }\end{array}$ \\
\hline 1 & 26520 & 38253 & 12 & 60 & 5693 & 35167 & 3 \\
\hline 2 & 30007 & 34015 & 11 & 61 & 15127 & 17652 & 9 \\
\hline 3 & 16797 & 21368 & 8 & 62 & 8131 & 28312 & 4 \\
\hline 4 & 26672 & 30291.5 & 12 & 63 & 18610 & 34785 & 9 \\
\hline 5 & 14346 & 10545 & 8 & 64 & 13311 & 31625 & 8 \\
\hline 6 & 12451 & 12267 & 6 & 65 & 18694 & 28158.5 & 9 \\
\hline 7 & 13787 & 8970 & 7 & 66 & 12153 & 29852 & 7 \\
\hline 8 & 19576 & 24437.8 & 12 & 67 & 16177 & 27424.7 & 8 \\
\hline 9 & 27895 & 37101 & 11 & 68 & 37270 & 36730 & 17 \\
\hline 10 & 21381 & 41022 & 12 & 69 & 30662 & 30590.9 & 16 \\
\hline 11 & 25302 & 34033 & 17 & 70 & 53255 & 45458.1 & 15 \\
\hline 12 & 14734 & 28380 & 8 & 71 & 20651 & 38303 & 10 \\
\hline 13 & 14203 & 15330 & 7 & 72 & 31419 & 31605 & 17 \\
\hline 14 & 14448 & 25255 & 8 & 73 & 22516 & 36927 & 10 \\
\hline 15 & 13279 & 30264 & 8 & 74 & 11361 & 21320 & 7 \\
\hline 16 & 20470 & 16574 & 9 & 75 & 11343 & 19491 & 6 \\
\hline 17 & 14619 & 28477 & 9 & 76 & 3988 & 9415 & 3 \\
\hline 18 & 16804 & 19708 & 8 & 77 & 5114 & 42314 & 4 \\
\hline 19 & 35453 & 50579 & 17 & 78 & 12620 & 22661 & 6 \\
\hline 20 & 20920 & 34308 & 12 & 79 & 13794 & 28796 & 8 \\
\hline 21 & 29883 & 47441.1 & 13 & 80 & 12696 & 18464 & 7 \\
\hline 22 & 29359 & 45070 & 13 & 81 & 17194 & 24411 & 6 \\
\hline 23 & 2445 & 1680 & 2 & 82 & 11820 & 24300 & 7 \\
\hline 24 & 21574 & 38851 & 12 & 83 & 13145 & 21364 & 5 \\
\hline 25 & 7338.7 & 21764 & 8 & 84 & 36704 & 56825 & 14 \\
\hline 26 & 19020 & 37101 & 11 & 85 & 8167 & 14338 & 5 \\
\hline
\end{tabular}

According to the method above, spatial variability is analyzed, model fitting is fulfilled and ordinary kriging is use to create interpolating surfaces. For example, the semi-variogram image of steam injection volume is shown as Fig. 1. In semi-variogram surface images, the center is zero distance of lag, and variability increase from low to high with colors from dark blue to green.

With ordinary kriging and mathematical fitting techniques, interpolating image of steam injection volume is shown as Fig. 2.

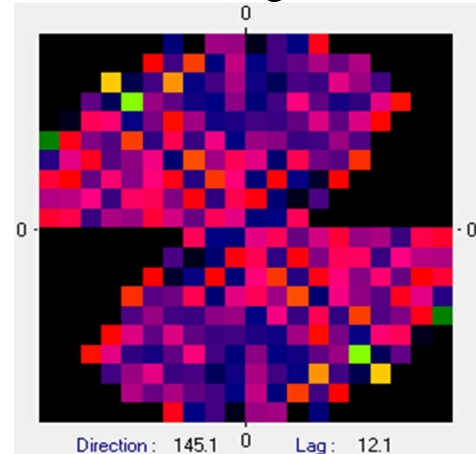

Fig. 1 Semi-variogram image

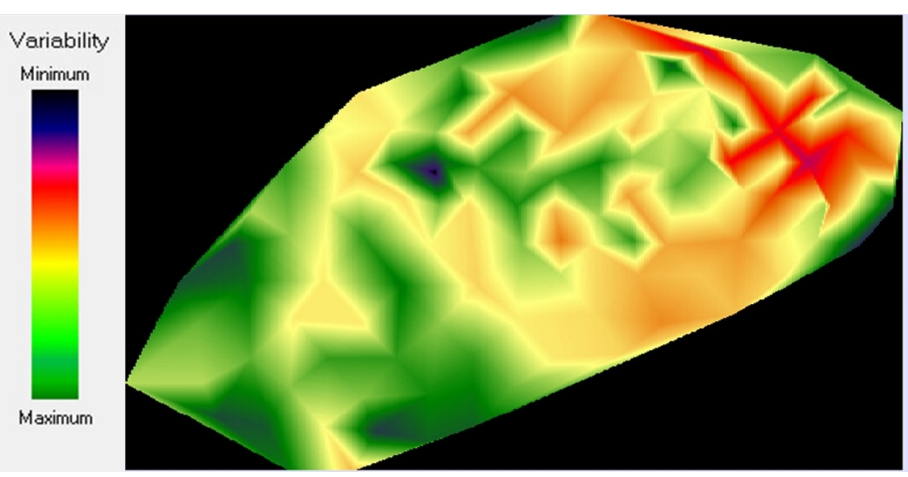

Fig. 2 Interpolating image of production volume 
With the same method, the semi-variogram image and interpolating image of other two factors, times of steam injection and volume of oil production, can be obtained. Before the calculation of failure risk based on above predictive model, those imagines need to be normalized. They can be standardized with fuzzy to 0 to 255 , in which 255 represents the maximum risk, and 0 represents the minimum risk. Then, the failure risk of underground casing pipe is worked out as Fig. 3.

\section{Results Analysis and Conclusions}

Through the construction of failure risk predictive model based on geostatistics, the failure risk of an application example for underground casing pipe is calculated. It can be found that the dangerous areas with more than 200 risk degree, and more attention is needed. Therefore, accurate prediction results can be obtained through this prediction model

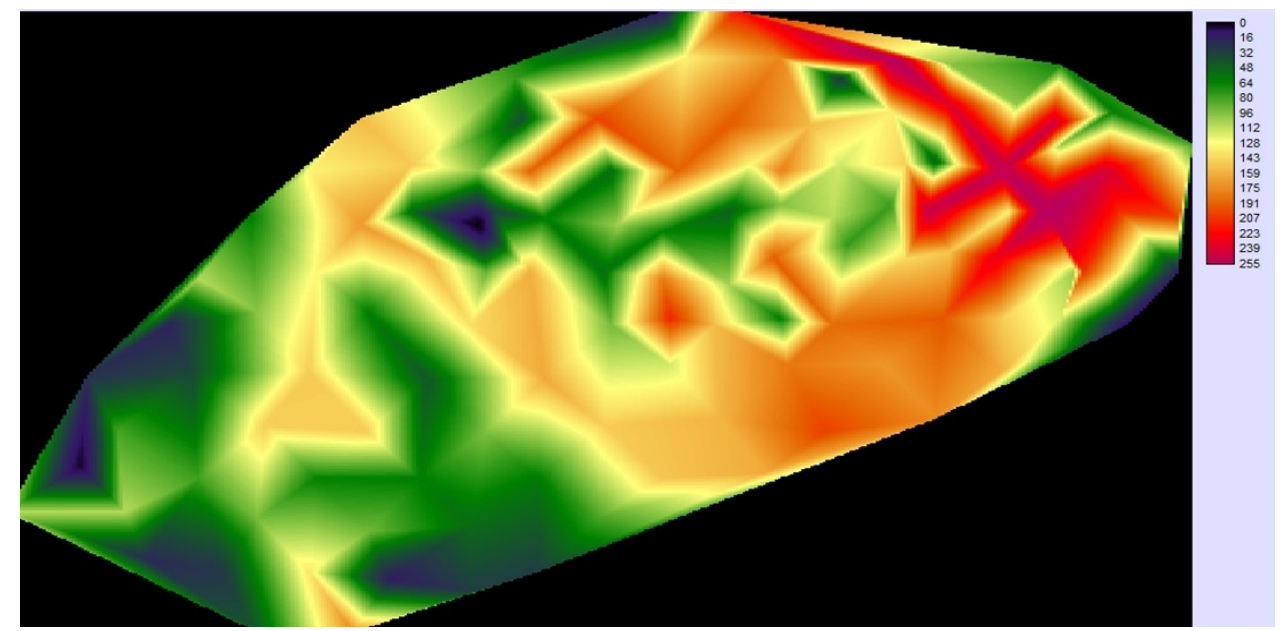

Fig. 3 Predictive result of application example

\section{Acknowledgements}

This research was financially supported by the China National Science Foundation (51378172), and Science Foundation of Hebei Province (E2014209089).

\section{References}

[1] W.M. Ryan, M.O. Joan and S. Daniel: Energy policy Vol. 35 (2007), p. 2151-2162

[2] T. Zhang, Q.J. Zhu, R. Ren, et al: Journal of Changzhou University (Natural Science Edition) Vol. 27 (2015), p. 51-55.

[3] H.Y. Guo, W.Y. Dong and M. Lou: Periodical of Ocean University of China Vol. 38 (2007), p. 503-507

[4] Q.J. Zhu, Y.H. Chen and L.Z. Jiang: Rock and Soil Mechanics Vol. 29 (2008), p. 2392-2396

[5] Z.C. Pei, Y.X. Liu, X.Q. Wang, et al: Northwestern Seismological Journal Vol. 27 (2005), p. 186-189

[6] A.Farkhutdinov, P. Goble, C.D. Fouquet, et al: Geothermics. Vol. 59 (2016), p. 56-66

[7] P.Goovaerts: Geostatistics for Natural Resources Evaluation (Oxford University Press, Oxford 1997).

[8] R.R. Yager: Information Sciences Vol. 178 (2008), p. 363-380 
[9] C.H. Wang, J.S. Yuan, C.Q. Jiang, et al: Journal of Anhui Agri. Sci. Vol.40(2012), p.10414-10418

[10] Q.J. Zhu, Y. Su, D.D. Wu: Int. J. Environment and Pollution Vol.42 (2010), p. 330-343

[11] W.B. Xu, Q.J. Zhu, H.B. Jia: Advanced Materials Research Vol. 807-809 (2013), p. 2595-2601

[12] Q.J. Zhu, B. Hejmanowska: Journal of Chemical and Pharmaceutical Research Vol. 5 (2013), p. 372-380. 